\title{
2,5-Dihydro-2,4,5-Trimethylthiazoline (TMT)-Induced Neuronal Activation Pattern and Behavioral Fear Response in GAD67 Mice
}

\author{
Kathrin Janitzky 1,2*, Olaf Prellwitz ${ }^{3}$, Herbert Schwegler', Yuchio Yanagawa5, \\ Thomas Roskoden ${ }^{4}$ \\ ${ }^{1}$ Department of Neurology, University of Magdeburg, Magdeburg, Germany \\ ${ }^{2}$ Leibniz Institute of Neurobiology, Magdeburg, Germany \\ ${ }^{3}$ Department of Orthopedics and Traumatology, Clinical Center in the Pfeiffersche Stiftungen, Magdeburg, \\ Germany \\ ${ }^{4}$ Institute of Anatomy, University of Magdeburg, Magdeburg, Germany \\ ${ }^{5}$ Department of Genetic and Behavioral Neuroscience, Gunma University, Maebashi, Japan \\ Email: *kathrin.janitzky@med.ovgu.de, o.prellwitz@gmail.com, herbert.schwegler@med.ovgu.de, \\ yanagawa@med.gunma-u.ac.jp, thomas.roskoden@med.ovgu.de
}

Received 22 June 2015; accepted 24 July 2015; published 27 July 2015

Copyright (C) 2015 by authors and Scientific Research Publishing Inc.

This work is licensed under the Creative Commons Attribution International License (CC BY).

http://creativecommons.org/licenses/by/4.0/

(c) (i) Open Access

\section{Abstract}

The synthetic predator odor 2,5-dihydro-2,4,5-trimethylthiazoline (TMT) was used to induce innate fear in male GAD67 mice and behavioral changes were correlated with c-fos mRNA levels as marker for neuronal activation to reveal underlying activated fear circuits. Results show the same amount of increased freezing and decreased rearing and grooming behavior of TMT-exposed GAD67 mice and wild type littermates, and therefore suggest that heterozygous knock-in of GFP in the GAD67 gene that is associated with a fifty percent decreased GAD67 protein level in the brain, has no impact on TMT-induced behavioral effects. Exposure to TMT significantly increased the number of c-fos mRNA positive cells in the main olfactory bulb (MOB), the lateral septum (LS), the bed nucleus of the stria terminalis (BNST), the central amygdala (CeA), the anterior-ventral (MeAav), the anterior-dorsal (MeAad) and the posterior-ventral (MeApv) part of the medial amygdala in GAD67 mice. Thus, to further investigate the role of GABAergic neurons in TMT-induced uncontrollable stress responses GAD67 mice that provide the advantage of prelabeled GABAergic neurons through the GABA neuron specific expression of GFP could be a suitable model organism.

\section{Keywords}

Predator Odor, 2,5-Dihydro-2,4,5-Trimethylthiazoline, c-fos mRNA In-Situ-Hybridization,

${ }^{*}$ Corresponding author.

How to cite this paper: Janitzky, K., Prellwitz, O., Schwegler, H., Yanagawa, Y. and Roskoden, T. (2015) 2,5-Dihydro-2,4,5Trimethylthiazoline (TMT)-Induced Neuronal Activation Pattern and Behavioral Fear Response in GAD67 Mice. Journal of Behavioral and Brain Science, 5, 318-331. http://dx.doi.org/10.4236/jbbs.2015.57032 


\section{Introduction}

Predator odors are particularly suited to induce stress in mice, as rodents are very sensitive to olfactory cues, and odors influence nearly every major class of rodent behaviour [1]. The perception of odors that indicate the presence and proximity of a predator elicits a vast spectrum of fear behavior that ranges from active behavior like fighting or fleeing up to passive behavior like freezing [2] [3]. Since the assessment of direct predation risk is important for the prey to avoid the direct encounter, perception and reaction to predator odors play a key role for small mammalian prey species while maximizing its chances for outliving the situation.

2,5-dihydro-2,4,5-trimethylthiazoline (TMT) is a chemically derived component of fox feces that induce robust innate fear and stress responses in laboratory mice and rats that were never exposed to a predator before [1] [4]-[7]. As TMT is a single molecule it circumvents problems of dosage and disposability linked with whole fecals or fur-derived predator odors. Due to these properties it has been widely used to investigate the influence of environmental conditions [7]-[9] and genetic dispositions [10]-[12] on innate fear behavior in rodents.

To investigate the role of GABAergic neurons in innate fear processes to TMT GAD67 mice might be a suitable model organism. In GAD67 mice enhanced green fluorescent protein (GFP) is expressed in GABAergic neurons under the control of the endogenous GAD67 gene promoter. GFP cDNA knock-in into the endogenous GAD67 gene leads to a decreased GAD67 protein level in the brain to half amounts as compared to wild type mice [13] [14]. GAD67 is the most relevant enzyme for stress-induced GABA synthesis, and a high proportion of BNST neurons express GAD67 [15]. Since the BNST is the most popular TMT-activated brain region in rodents, and acute stress was shown to potentially increase GAD67 expression of BNST neurons [16], an intact GABA response within the BNST appears to be critical for TMT-induced innate fear behavior. Hence, we suggest that is should be ruled out that decreased GAD67 levels in GAD67 mice affect TMT stress-related behavior before using these mice as a model for detailed study of GABAergic neural circuits important for TMT-related innate fear.

To rule out that heterozygous insertion of the GFP protein to the GAD67 gene affects behavioral reaction to TMT, in the present study we directly compared behavioral responses of transgenic GAD67 mice with those of their wild type littermates (genetic background C57BL/6J). Butyric acid (BA) and diethylphthalate (DEP) were used as control odors, because DEP was applied as a solvent to dilute TMT in prior studies [17], and BA was a pungent odor that activated the trigeminal system [18].

Afterwards we conducted c-fos mRNA in-situ-hybridization with the brains of GAD67 mice. Since we have already published detailed investigation of TMT-activated neural circuits in C57BL/6J wild type mice using Fos immunohistochemistry [19] and prior real time PCR data of our group showed no differences in TMT-induced c-fos mRNA levels (in the ventral and dorsal olfactory bulbs, the amygdala, the BNST and the PVN) between GAD67 mice and their wild type littermates (unpublished data), wild type mice were not analyzed in this study. c-fos mRNA encodes the FOS protein that is an immediate-early-gene expressed rapidly and transiently after increased neuronal activation [20]-[22]. Therefore, the number of c-fos mRNA positive cells was analyzed for the identification of regions with increased neuronal activity during TMT exposure [5] [23]-[27]. TMT-induced c-fos mRNA expression was analyzed in the MOB, the AOB, the medial and the central nucleus of the amygdala as well as in the BNST and the lateral septum, structures known to be central for TMT-induced behavioral responses [1] [5] [24] [27]-[44]. Therefore, the present study provides the basis to further investigate the impact of GABAergic neurons in limbic structures for TMT-induced innate fear using GAD67 mice that provide the advantage of prelabeled GABAergic neurons through the GABA neuron specific expression of GFP.

\section{Results and Statistical Analyses}

\subsection{Behavioral Analyses}

\subsubsection{Genotype}

T-tests comparing behavioral response towards each odor reveal no significant influence of genotype on behavioral responses to odor exposure (Figures 1(a)-(c)). 


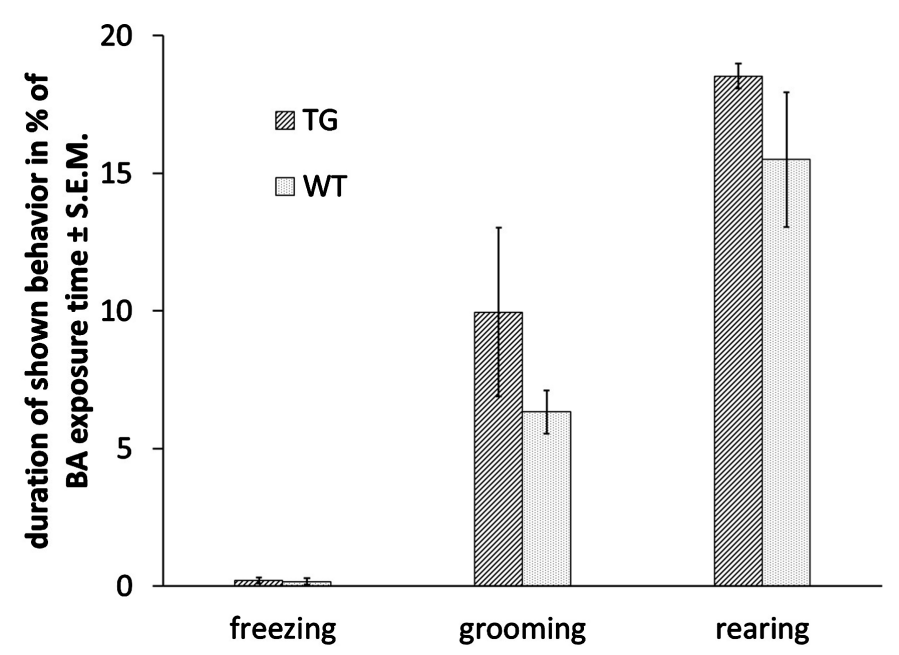

(a)

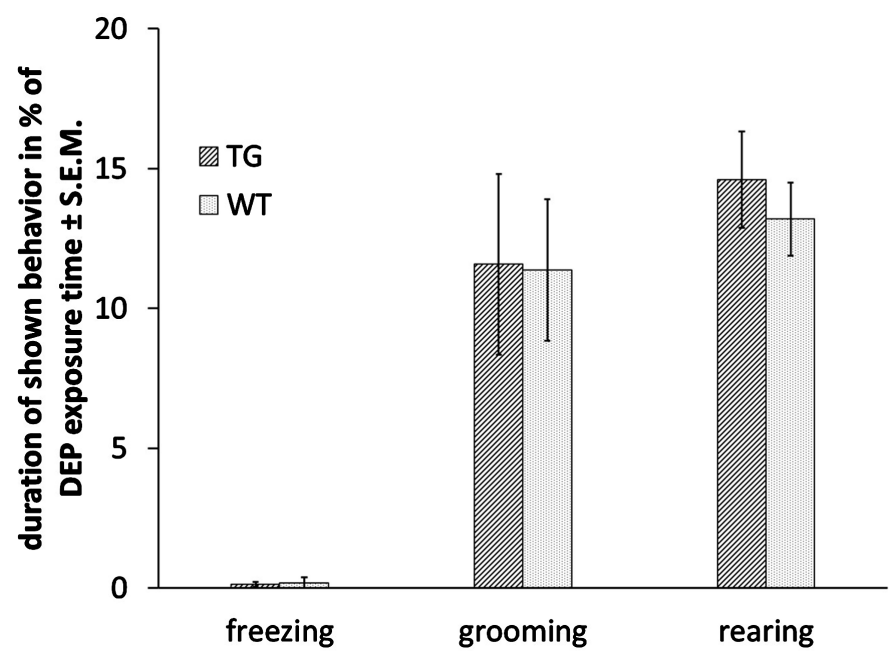

(b)

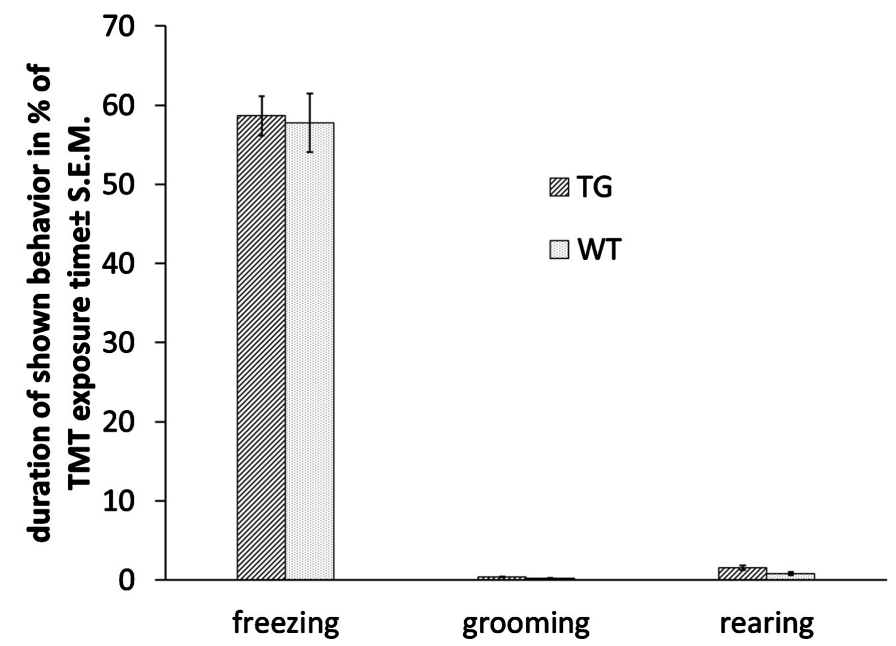

(c)

Figure 1. Duration of time spent freezing, grooming and rearing shown by GAD67 (TG) mice and wild type (WT) littermates exposed to (a) butyric acid (BA); (b) diethylphthalate (DEP) or (c) 2,5-dihydro-2,4,5,-trimethylthiazoline (TMT). No significant genotype differences were found. 


\subsubsection{Freezing}

ANOVA indicates a strong effect of odor exposure on freezing behavior $(\mathrm{F}=780.54$; $\mathrm{df}=2.24 ; p<0.0001$; Figure 2(a)). Tukey's HSD post-hoc test reveals that freezing is significantly increased during TMT exposure ( $p$ $<0.0001)$. Exposure to BA did not significantly change freezing behavior compared to DEP exposure $(p>0.9)$. One-way ANOVA reveals an effect of the duration of TMT exposure $(\mathrm{F}=71.69 ; \mathrm{df}=2.24 ; p<0.0001$; Figure 2(b)). Tukey's HSD post-hoc test shows significantly increased freezing time comparing interval 1 (min. 2 - 10) with 2 (min. 11 - 20) and 3 (min. 21 - 30) $(p<0.0001$; Figure 2(b)). Stereotypical freezing reaction to TMT exposure peaks after 10 minutes and was unchanged for the next 20 minutes.

\subsubsection{Grooming}

ANOVA indicates a significant effect of odor exposure on grooming behavior $(\mathrm{F}=15.72 ; \mathrm{df}=2.24 ; p<0.0001$; Figure 2(a)). Tukey's HSD post-hoc test reveals excessively decreased grooming behavior during TMT exposure ( $p<0.0001$ compared to DEP exposure; $p<0.01$ compared to BA exposure). Time spent grooming during DEP and BA exposure was not significantly different. The effect of TMT exposure on grooming behavior was also time dependent $(\mathrm{F}=25.56$; $\mathrm{df}=2.24 ; p<0.0001)$. During the first 10 minutes of TMT exposure animals

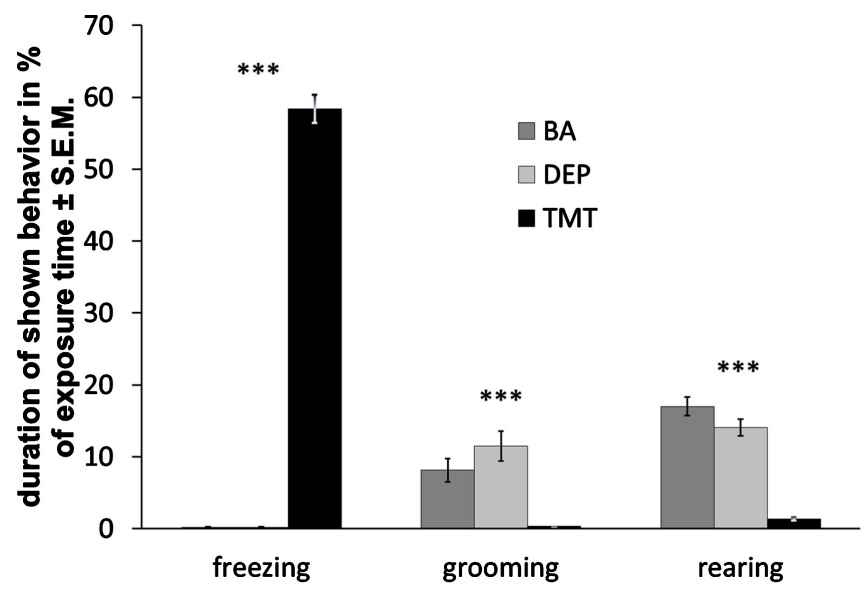

(a)

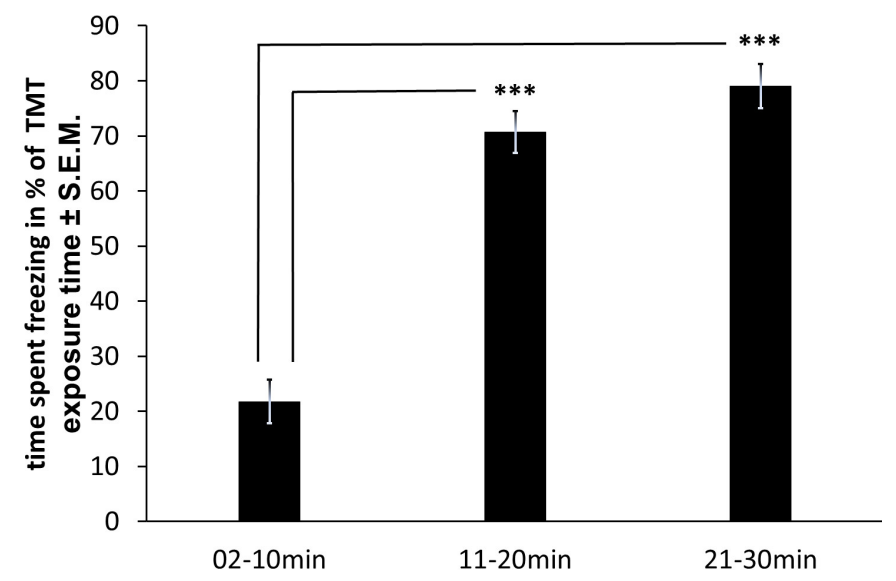

(b)

Figure 2. (a) Duration of time spent freezing, grooming and rearing in percent of time \pm S.E.M. shown by mice exposed to butyric acid (BA), diethylphthalate (DEP) or 2,5-dihydro-2,4,5-trimethylthiazoline (TMT). A one-way ANOVA reveals a significant exposure effect on all three behaviors. TMT exposure significantly increased the time spent freezing and decreased grooming and rearing, while behavior shown by mice exposed to DEP or BA was not significantly different (bars represent mean time in percent \pm S.E.M.; ${ }^{* * *} p<0.0001$ ); (b) Time spent freezing during TMT exposure. There was a significant increase of freezing behavior comparing the first ten minutes with the second and third ten minutes of TMT exposure (bars representing mean time in percent \pm S.E.M.; ${ }^{* * *} p<0.0001$ ). 
showed significantly more grooming than in interval $2(p<0.0001)$ and $3(p<0.0001$; data not shown). Compared to controls TMT-exposed animals showed significantly less grooming even during the first 10 minutes of the experiment.

\subsubsection{Rearing}

ANOVA indicates a significant effect of odor exposure on rearing behaviour $(\mathrm{F}=54.01 ; p<0.0001$; $\mathrm{df}=2.24$; Figure 2(a)). Tukey's HSD post-hoc test reveals a significant decrease of rearing behavior during TMT exposure as compared to DEP and BA exposure ( $p<0.0001$; each). In between the DEP and BA exposure groups no significant difference in rearing behavior was found $(p>0.1)$.

The effect of TMT exposure on rearing behavior was also dependent on the duration of odor exposure $(\mathrm{F}=$ 24.38; $\mathrm{df}=2.24 ; p<0.0001)$. In interval 1 significantly more rearing was detected than in interval 2 and 3 ( $p<$ 0.0001; data not shown).TMT-exposed mice showed significantly less rearing even during the first 10 minutes of the experiment compared to controls.

\section{2. c-fos mRNA In-Situ-Hybridization}

Student's t-test shows no significant differences between the numbers of c-fos mRNA positive cells of DEP- and BA-exposed GAD67 mice. Hence, data of DEP- and BA-exposed mice were pooled and tested against TMTexposed GAD67 mice.

Student's t-test reveals significant c-fos mRNA induction in themain olfactory bulb (MOB) of TMT-exposed mice $(p<0.05$; Figure 3(a) and Figure 4(a)). In detail significantly more c-fos mRNA positive cells were found in the glomerular zone (MOBgl) $(p<0.05$; Figure 3(b) and Figure 4(a); Table 1), the mitral cell layer

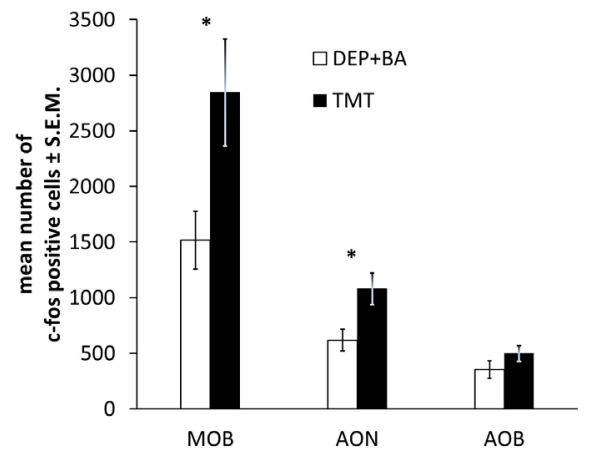

(a)

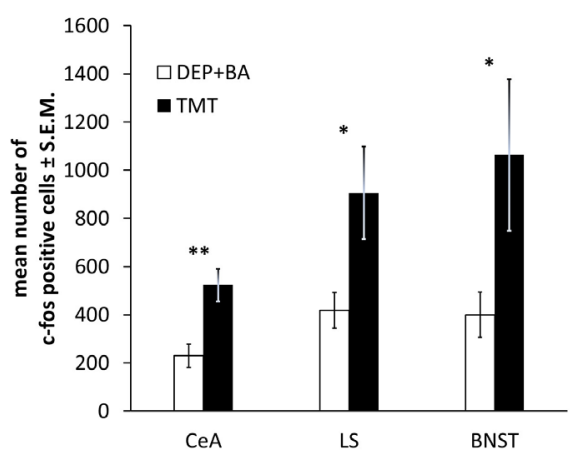

(c)

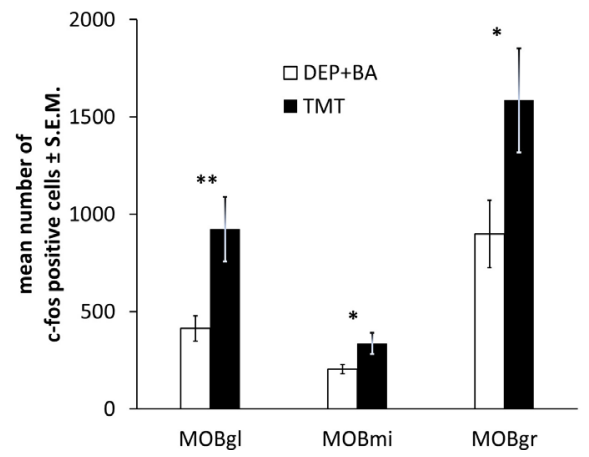

(b)

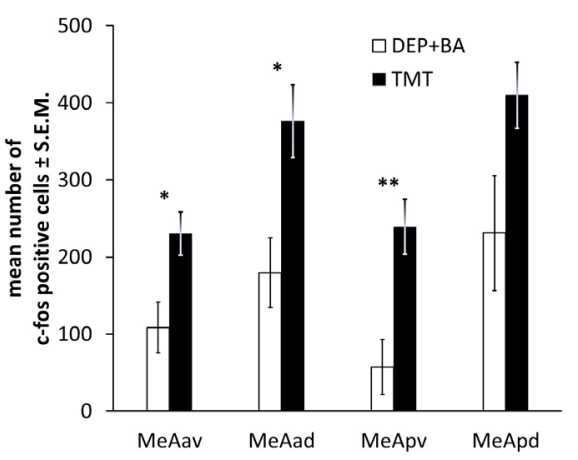

(d)

Figure 3. c-fos mRNA in-situ-hybridization: Mean number of c-fos mRNA positive cells \pm S.E.M. in diethylphthalate (DEP)- and butyric acid (BA)-exposed vs. 2,5-dihydro-2,4,5-trimethylthiazoline (TMT)-exposed GAD67 mice. (a) In the main olfactory bulb (MOB), the anterior olfactory nucleus (AON) and the accessory olfactory bulb (AOB); (b) In the MOB: the glomerular (MOBgl), the mitral (MOBmi) and the granular cell layer (MOBgr); (c) In the central amygdala (CeA), the lateral septum (LS), the bed nucleus of the stria terminalis (BNST); (d) In the medial amygdala (MeA): the anterior-ventral (MeAav), the anterior-dorsal (MeAad), the posterior-ventral (MeApv) and the posterior-dorsal (MeApd) parts (bars represent mean numbers \pm S.E.M.; $\left.{ }^{* *} p<0.01 ;{ }^{*} p<0.05\right)$. 


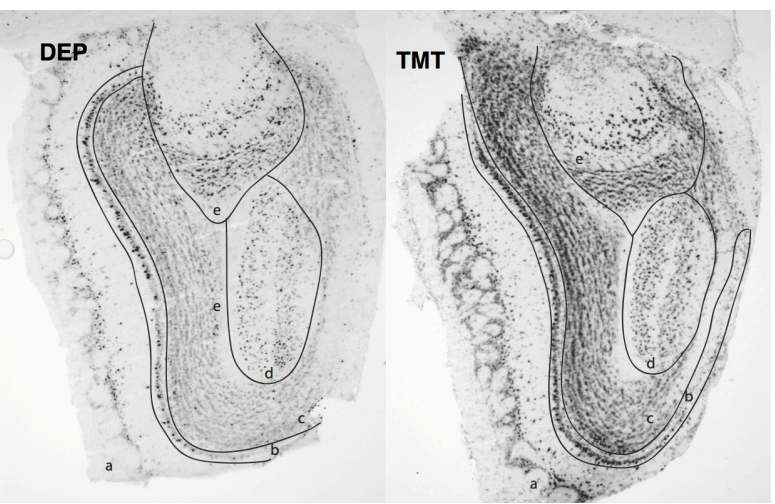

(a)

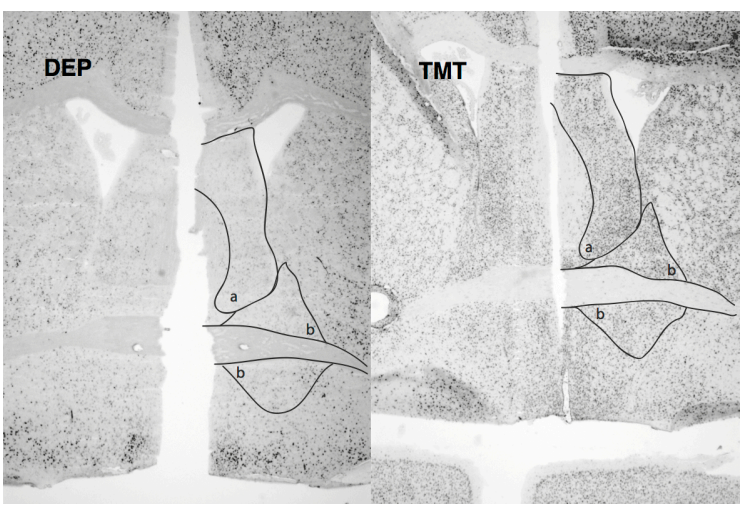

(b)

Figure 4. Microphotographs of in situ hybridization slices. Expression of c-fos mRNA (a) in the main olfactory bulb (MOB) und accessory olfactory bulb (AOB) of diethylphthalate (DEP)- and 2,5-dihydro-2,4,5-trimethylthiazoline (TMT)-exposed mice a: glomerular layer of the MOB; b: mitral cell layer of the MOB; c: granular cell layer of the MOB; d: anterior olfactory nucleus; e: accessory olfactory bulb); (b) Expression of c-fos mRNA in a: the lateral septum (LS) and b: the bed nucleus of the stria terminalis (BNST) of DEP- und TMT-exposed mice.

Table 1. c-fos mRNA in-situ-hybridization: Mean number of c-fos mRNA positive cells \pm S.E.M. in diethylphthalate (DEP) and butyric acid (BA)-treated vs. 2,5-dihydro-2,4,5-trimethylthiazoline (TMT)-treated GAD67 mice, ${ }^{* *} p<0.01 ;{ }^{*} p<0.05$.

\begin{tabular}{|c|c|c|c|}
\hline Brain regions & $\begin{array}{c}\text { DEP/BA exposure } \\
\text { (mean cell counts } \pm \text { SEM) }\end{array}$ & $\begin{array}{c}\text { TMT exposure } \\
\text { (mean cell counts } \pm \text { SEM) }\end{array}$ & $P$ for $>t$ \\
\hline Main olfactory bulb & $506 \pm 86$ & $948 \pm 160$ & $0.02^{*}$ \\
\hline Main olfactory bulb glomerular cell layer & $413 \pm 65$ & $923 \pm 166$ & $0.006^{*}$ \\
\hline Main olfactory bulb mitral cell layer & $205 \pm 23$ & $336 \pm 54$ & $0.02^{*}$ \\
\hline Main olfactory bulb granular cell layer & $899 \pm 174$ & $1585 \pm 267$ & $0.05^{*}$ \\
\hline Accessory olfactory bulb & $354 \pm 79$ & $499 \pm 70$ & 0.27 \\
\hline Anterior olfactory nucleus & $618 \pm 98$ & $1080 \pm 142$ & $0.02^{*}$ \\
\hline Lateral septum & $419 \pm 75$ & $906 \pm 193$ & $0.02^{*}$ \\
\hline Bed nucleus of the stria terminalis & $400 \pm 95$ & $1063 \pm 315$ & $0.02^{*}$ \\
\hline Periventricular hypothalamic nucleus & $24 \pm 7$ & $129 \pm 80$ & 0.08 \\
\hline Paraventricular hypothalamic nucleus & $115 \pm 21$ & $192 \pm 26$ & 0.056 \\
\hline Central nucleus of the amygdala & $230 \pm 48$ & $523 \pm 67$ & $0.02^{*}$ \\
\hline Medial nucleus of the amygdala anterodorsal part & $179 \pm 45$ & $376 \pm 47$ & $0.02^{*}$ \\
\hline Medial nucleus of the amygdala anteroventral part & $108 \pm 33$ & $230 \pm 28$ & $0.04^{*}$ \\
\hline Medial nucleus of the amygdala posterodorsal part & $231 \pm 75$ & $410 \pm 43$ & 0.14 \\
\hline Medial nucleus of the amygdala posterventral part & $57 \pm 36$ & $239 \pm 36$ & $0.001^{*}$ \\
\hline
\end{tabular}

(MOBmi) ( $p<0.05$; Figure 3(b) and Figure 4(a); Table 1) and the granular cell layer (MOBgr) $(p=0.05$; Figure 3(b) and Figure 4(a); Table 1) of the MOB. Furthermore, significant c-fos mRNA induction was detected in the anterior olfactory nucleus (AON) ( $p<0.05$; Figure 3(a) and Figure 4(a); Table 1), but no significantly changed number of c-fos mRNA positive cells were found in the accessory olfactory bulb (AOB) $(p>0.2$; Figure 3(a) and Figure 4(a); Table 1) of TMT-exposed GAD67 mice compared to controls. Results indicate processing of TMT by the MOB and no relevant input of the AOB. 
Moreover significant c-fos mRNA induction was found in the lateral septum (LS) ( $p<0.05$; Figure 3(c) and Figure 4(b); Table 1) and the bed nucleus of the stria terminalis (BNST) $(p<0.05$; Figure 3(c) and Figure 4(b); Table 1) of TMT-exposed GAD67 mice compared to controls.

Detailed analyses of subnuclei of the amygdala reveal significant c-fos mRNA induction in the central nucleus of the amygdala (CeA) $(p<0.01$; Figure 3(c); Table 1$)$ and in subnuclei of the medial nucleus of the amygdala (MeA) of TMT-exposed GAD67 mice compared to controls. Significantly more c-fos mRNA positive cells were found in the anterodorsal medial nucleus of the amygdala (MeAad) $(p<0.05$; Figure 3(d); Table 1), the anteroventral medial nucleus of the amygdala (MeAav) $(p<0.05$; Figure 3(d); Table 1), and the posteroventral medial nucleus of the amygdala (MeApv) $(p<0.05$; Figure 3(d); Table 1$)$ of TMT-exposed mice. Whereas in the posterodorsal medial nucleus of the amygdala (MeApd) ( $p>0.1$; Figure 3(d); Table 1) no significant differences of c-fos mRNA positive cells were found in TMT-exposed GAD67 mice compared to controls.

A trend towards an increase of c-fos mRNA was found in the paraventricular hypothalamic nucleus (PVN) ( $p$ = 0.056; Table 1), indicating a tendentially effect of TMT exposure on the hypothalamic-pituitary-adrenal (HPA) axis and consecutive TMT-induced neuroendocrine responses. No significant c-fos mRNA induction was found in the periventricular hypothalamic nucleus PV ( $p>0.05$; Table 1). A survey of all findings gives Table 1.

\section{Discussion}

Results of the present study show that behavioral responses to TMT are unaffected by decreased GAD67 protein levels in the brain caused by the loss of one copy of the GAD67 gene in male GAD67 mice, as indicated by the same amount of increased freezing behavior and decreased grooming and rearing behavior of TMT-exposed GAD67 mice and wild type littermates. Hence, GAD67 mice might be a suitable model organism to further investigate the role of GABAergic neurons in TMT-induced innate fear behavior.

In-situ-hybridization studies in GAD67 mice show TMT-induced significant increase of c-fos mRNA positive cells in the glomerular (MOBgl), the mitral (MOBmi) and the granular cell layer (MOBgr) of the main olfactory bulb, the lateral septum (LS), the bed nucleus of the stria terminalis (BNST) and the central amygdala (CeA). Analyses of subnuclei of the medial amygdala (MeA) indicate TMT-induced activation of the anterior-ventral (MeAav), the anterior-dorsal (MeAad) and the posterior-ventral (MeApv) parts of the MeA. Thus, present results confirm already published data of TMT-induced Fos expression in C57BL/6J wild type mice (Janitzky et al., 2015). Hence, data suggest that neuronal activation of these nuclei may be functionally relevant for TMT-induced innate fear behavior in male GAD67 mice.

As the behavioral reaction to TMT depends on the genetic background [10]-[12], our present results, showing comparable behavioral responses of GAD67 mice and their wild type littermates, support GAD67 mice as a suitable model organism to further study the role of GABAergic neurons for innate fear and uncontrollable stress responses to TMT in these mice.

TMT is a synthetically derived component of fox feces [6] that offers the advantages of high-precision and reproducible exposure. Hence, we used it as a predator odor stimulus to elicit innate fear and uncontrollable stress responses [4] [5] [7] [45]-[48]. Our results show strong TMT-induced increase of freezing behavior and decrease of grooming and rearing behavior in male GAD67 mice. Behavioral effects of GAD67 mice are similar to those of their wild type littermates and therefore present results reveal that TMT is suitable to induce innate fear and uncontrollable stress responses in GAD67 mice characterized by half amounts of GAD67 protein level in the brain [13] [14].

Subsequently, c-fos mRNA expression was detected in several olfactory and fear-related brain sites of GAD67 mice to investigate responsible brain circuits using in-situ-hybridization. Analyses of c-fos mRNA expression in olfactory brain sites show a significant increase of c-fos mRNA positive cells in the glomerular (MOBgl), the mitral (MOBmi) and the granular cell layer (MOBgr) of the main olfactory bulb (MOB). Whereas, no significant increase of c-fos mRNA positive cells was found in the accessory olfactory bulb (AOB) of TMT-exposed GAD67 mice compared to controls that were exposed to the pungent odor BA or the odorless control substance DEP. These findings are in accordance with prior own results and the results of other groups and imply that TMT is perceived and processed by the MOB [5] [24] [27] [35] [38].

Since, previous studies showed that the medial nucleus of the amygdala $(\mathrm{MeA})$ receives both direct and indirect projections from the olfactory system [49]-[52] our present results indicate that olfactory detection of TMT 
depends on the MOB and that further processing of TMT-related information may involve a MOBgl-MeA-projection. More particularly the anterodorsal (MeAad), the anteroventral (MeAav) and the posteroventral parts of the medial nucleus of the amygdala (MeApv) show significant TMT-induced increase of c-fos mRNA positive cells contrary to the posterodorsal part of the medial nucleus of the amygdala (MeApd). Results are in line with prior studies showing that inactivation of the MeA dramatically impaired unconditioned freezing to TMT in rats [32], which was not attributable to a deficient olfactory detection [37] or a global deficit in conditioned fear behavior to other stimuli than predator odors [52] [53]. Significant TMT-induced activation of the MeAad was supported by findings of other groups indicating reliable TMT-evoked c-fos mRNA induction in this nucleus [24]. Contrary to TMT, cat collar odor was shown to activate the posterior parts of the medial nucleus of the amygdala, called "vomeronasal amygdala", while getting main projections from the AOB [29]. Hence, observed differences of MeA activation may be related to differential olfactory processing of TMT versus cat collar predator odor stimuli. Detection of cat collar odor seems to be mediated by the vomeronasal apparatus [29]. Whereas, TMT activates two sets of glomeruli, one located in the dorsal and the other located in the ventral domain of the MOB [35]. Kobayakawa and colleagues proposed that innate fear responses to TMT depend on a neuronal pathway arising from the dorsal domain of the main olfactory bulb that activates the bed nucleus of the stria terminalis (BNST). The BNST in turn was thought to stimulate the hypothalamic-pituitary-adrenal (HPA) axis [35]. Contrary to the established role of the BNST for unconditioned innate fear, the CeA is a region accepted as central for conditioned fear [5] [54]-[56]. Therefore, present results indicating significant TMT-induced neuronal activation of the CeA appear to be in conflict with this suggestion and with data demonstrating that inactivation of the CeA is ineffective in attenuating unconditioned freezing behavior to TMT [32]. But other groups also found a significant TMT-induced activation of the CeA [24] [55], and assume that an activation of this nucleus dependent on the exposure environment might be possible [37] [40] [57]. In summary it can be said, that the role of the CeA for behavioral responses to TMT predator odor is still uncertain, but the central role of the MeA for the processing of TMT-related information and subsequent TMT-induced fear behavior seems likely on the basis of the present results.

Significantly increased c-fos mRNA expression in the BNST and the lateral septum (LS) of TMT-exposed mice support the theory that these structures are part of a TMT-activated innate fear circuit [30]. As GABAergic neurons are the main type in the BNST and most expressing GAD67 [15] [58], decreased GAD67 protein levels might have modify TMT stress-induced anxiety-like behavior in GAD67. Given the fact that TMT-induced innate fear behavior was unimpaired in GAD67 mice as indicated by the same amounts of increased freezing and decreased grooming and rearing behavior compared to their wild type littermates, these mice seem to be applicable to study the role of GABAergic neurons in the BNST for TMT-induced innate fear.

Data support that innate fear responses to TMT involve genetically programmed and hard-wired neural circuits. These innate evolutionary conserved fear pathways seem to include strongly connected limbic structure like the MeA and the BNST that both receive direct olfactory information and send projections to the PVN and brain stem structures allowing rapid activation of the HPA stress axis and immediate initiation of behavioral reactions after perception of predator odor for outliving the situation. Present and prior data of our group as well as results of other investigators indicate an important role of the BNST for innate fear responses to TMT [5] [24] [32] [35] [55] [56]. Since, pharmacological inhibition of the BNST (but not the amygdala) by the GABA-A receptor agonist muscimol was shown to disrupt fear responses to TMT [32] [54], and since, increased anxiety levels were reported after inactivation of GABA synthesis within the BNST [59], it can be assumed that GABAergic mechanisms in the BNST are involved in TMT-induced innate fear responses. It was already shown that GABAergic BNST neurons can inhibit the HPA axis via direct projections to the PVN [58], and by the way of GABA-GABA disinhibitory connections [60], the amygdala is in a position to cause a stress-induced activation of the HPA axis. Since, stressful events, like the exposure to a predator odor, are able to activate GABAergic neurons within the BNST [61] a delivering of the BNST from the inhibitory GABAergic influence of the amygdala can be supposed.

Therefore, the present results that show an unimpaired TMT-induced fear behavior of GAD67 mice indicate them as a suitable mouse model to further investigate GABAergic mechanisms within the BNST for our better understanding of neural circuits relevant for innate fear responses [32] [42] [43] [62]. Since, the BNST is the most popular TMT-activated brain region in mice [5] [19] [56], and BNST neurons are regulated by an extensive network of GABAergic neurons [56] [59] [60] [63], such a research seems to be interesting. 


\section{Materials and Methods}

\subsection{Test for Behavioral Changes}

\subsubsection{Subjects}

Sixteen adult male GAD67 mice and ten adult male wild type littermates (C57BL/6J background strain), both 12 - 20 weeks of age, were used in the present experiment. GAD67 mice provide the advantage of prelabeled GABAergic neurons through the GABA neuron specific expression of GFP. Insertion of the gene encoding for GFP under the control of the GAD67 promotor induces a loss of one copy of the GAD67 gene. Heterozygous GAD67 mice were identified by PCR analysis of tail probes, using primers specific for the GFP sequence (TRGFP-8: CTGCTTGTCGGCCATGATATAGACG; TR-16: GGCACAGCTCTCCCTTCTGTTTGC; TR-3: GCTCTCCTTTCGCGTTCCGACAG).

All animals were housed in groups from 2 to 5 in standard cages, maintained a 12 hour light-dark-cycle (lights on at $7 \mathrm{AM}$ ) and food and water ad libitum. Mice were deeply anaesthetized with 0.4 ml Narcoren (Merial $\mathrm{GmbH}$, Hallbergmoos, Germany) i.p. after odor exposure before decapitation. The dissected brains were shockfrozen in $-40^{\circ} \mathrm{C}$ cold 2-methylbutane (Carl Roth $\mathrm{GmbH}$ und $\mathrm{Co} \mathrm{KG}$, Karlsruhe, Germany) and stored at $-40^{\circ} \mathrm{C}$ until further processing.

All procedures were committed by the European Council guideline 86/609/EEC and approved by local authorities.

\subsubsection{Odor Exposure}

Sixteen GAD67 mice (6 DEP-, 4 BA- and 6 TMT-exposed) and ten C57BL/6J (3 DEP-, 4 BA- and 3 TMT-exposed) were exposed to either diethylphthalate (DEP) (Sigma-Aldrich, Taufkirchen, Germany), butyric acid (BA) (Sigma-Aldrich, Taufkirchen, Germany) or 2,5-dihydro-2,4,5-trimethylthiazoline (TMT) (PheroTech, Delta, Kanada). Mice were distributed to the exposure groups in pseudo-randomized way in order to gain a homogenous contingent of genotypes and ages in each group.

\subsubsection{Apparatus for Odor Exposure}

Odor exposure took place in a box made of grey PVC $(30 \times 30 \times 30 \mathrm{~cm})$ with a plug-in board allowing the insertion of pieces of filter paper (Macherey-Nagel, Düren, Germany). The top of the box was made of clear plexiglas ${ }^{\circledR}$ to allow videotaping with a camera (Carl Zeiss AG, Jena, Germany) mounted above.

\subsubsection{Procedure}

All experiments were performed between 8 a.m. and 1 p.m. at low light conditions. Before testing, mice were familiarized with the odor exposure environment on two consecutive days. During the habituation sessions that lasted 30 minutes filter papers without odor were used.

To avoid any odor interference only one odorant a day was tested and the exposure box was cleaned after each animal using Descosept AF (Dr. Schumacher GmbH, Melsungen, Germany) and paper tissues (Carl Roth GmbH und Co KG, Karlsruhe, Germany). The animals were brought separately in clean cages from the housing area to the experimental room, where they were placed into the odor exposure box. The test procedure took 30 minutes. After the first minute a piece of filter paper with $35 \mu \mathrm{DEP}$, BA or TMT was placed into the box via the plug-in board. Since c-fos mRNA peaks 30 minutes after stimulus onset, odor exposure lasted 30 minutes [33] and mice were sacrificed immediately thereafter to minimize side effects of post exposure handling on neuronal activation pattern.

\subsubsection{Behavioral Analyses}

The videotapes were analyzed with regard to defensive behavior, i.e. freezing and non-defensive behavior, i.e. grooming and rearing. Freezing was defined as absence of any movement except for respiratory-related movements. The time the animal spent on its hind limbs exploring the environment was counted as rearing.

For this purpose the time of the experiment was divided into 4 time intervals:

Interval 0: minute 1 (no odor exposure).

Interval 1: minutes 2 - 10.

Interval 2: minutes 11 - 20.

Interval 3: minutes 21 - 30. 


\subsection{Histology}

To detect increased neuronal activation in olfactory and fear-related brain sites we performed c-fos mRNA insitu-hybridization with the brains of GAD67 mice (4 DEP-, 4 BA- and 4 TMT-exposed).

\subsection{1. c-fos mRNA In-Situ-Hybridization (ISH)}

Therefore, the deeply frozen brains were cut on a cryostat (Leica Microsystems, Wetzlar, Germany) into $16 \mu \mathrm{m}$ thick coronary sections. Every fourth slice corresponding to the Figures 01-07 and 20-47 in Franklin's Atlas of the Mouse Brain (Franklin and Paxinos, 2008) was mounted alternating on bare and silanized glass slides for later ISH or Nissl staining. c-fos 1 DNA cloned in the vector pSP65 (Promega, Madison, USA) was kindly allocated by the Institute of Physiology of the Otto von Guericke University Magdeburg. XL blue MRF (Stratagene, Amsterdam, Netherlands) cells were transfected with pSP65 plasmid using the Transform Aid Bacterial Transformation Kit (Fermentas, St. Leon-Rot, Germany). The transfected bacteria were streaked on a selective medium (lysogeny-broth and ampicilline). After preparing the plasmid using QIAprep Spin Miniprep Kit (Qiagen, Hilden, Germany) restrictive digestion was done using BamH1 (Fermentas, St. Leon-Rot, Germany). The products were isolated by gel electrophoresis and the amplified c-fos1 DNA was extracted from the gel using QIA quick Gel Extraction Kit (Qiagen, Hilden, Germany) and transcribed into sense- and anti-sense mRNA by SP6RNA-Polymerase (Roche Diagnostics GmbH, Mannheim, Germany) using digoxigenin-labeled desoxyribonucleotide triphosphates (Roche Diagnostics GmbH, Mannheim, Germany). After ethanol (Carl Roth GmbH und Co KG, Karlsruhe, Germany) precipitation the c-fos mRNA probes were ready to use. The sense probe was used for testing if there were any unspecific RNA-RNA interactions. No unspecific labelling with the sense probe occurred. Hence, the anti-sense probe was used for further procedure to identify c-fos mRNA positive cells. For the localization of the c-fos mRNA hybrids washed slices were incubated with an antibody against digoxigenin (Roche Diagnostics GmbH, Mannheim, Germany) coupled with alkaline phosphatase (AP). After addition of 5Brom-4-Chlor-3-Indolyl-Phosphat/Nitro Tetrazolium Blue Chloride) (BCIP/NBT) the AP produces a brown colorant near the RNA-RNA hybrids.

\subsubsection{Nissl Staining}

Nissl staining was done using a modified protocol and Cresylviolett (Dr. Th. Schuchardt, München, Germany) as colorant.

\subsubsection{Cell Counts}

c-fos mRNA positive cells were counted in one hemisphere of the following brain regions: the main olfactory bulb glomerular zone (MOBgl), the main olfactory bulb mitral cell layer (MOBmi), the main olfactory bulb granular cell layer (MOBgr), the anterior olfactory nucleus (AON), the accessory olfactory bulb (AOB), the lateral septum (LS), the bed nucleus of the stria terminalis (BNST), the central nucleus of the amygdala (CeA), the anterodorsal medial nucleus of the amygdala (MeAad), the anteroventral medial nucleus of the amygdala (MeAav), the posterodorsal medial nucleus of the amygdala (MeApd), the posteroventral medial nucleus of the amygdala (MeApv), paraventricular hypothalamic nucleus (PVN) and the periventricular hypothalamic nucleus.

c-fos mRNA positive cells were counted at hundredfold magnification using a camera lucida (Leitz GmbH, Wetzlar, Germany). The brain areas in question were identified using the Nissl-stained sections and the Allen Reference Atlas for C57BL/6J mice (Dong, 2008). After outlining the region of interest the Nissl-stained sections were replaced by the corresponding in-situ-hybridized sections and all c-fos mRNA positive cells lying within the marked region were counted. Because of different amounts of slices per region the mean number of cells per region was calculated and used for further statistics.

These counts were carried out by overlaying a $100 \times 100 \mu \mathrm{m}$ grid (ImageJ1.46r, NIH, USA) over microphotographs (Carl Zeiss AG, Jena, Germany) and counting all c-fos positive cells within one square in three sequent slices. Comparative cell-counts were performed in layer two of the secondary somatosensory cortex (S2) in order to test whether there was a general neuronal activation caused by TMT exposure.

\subsection{Statistics}

To test whether there was an influence of genotype on behavior wild type (WT) and transgenic mice (TG) were compared using unpaired Student's t-tests for each exposure group. Collected behavioral data (freezing, groom- 
ing, and rearing) were analyzed by one-way ANOVA with odor exposure as factor. For further analyses Tukey’s HSD post-hoc test was performed in order to find out which odor was responsible for the alterations being significant in the ANOVA. Additionally, a one-way ANOVA followed by Tukey's HSD post-hoc test was carried out for TMT exposure and each odor using the time interval as factor.

Counts of c-fos mRNA positive cells were analyzed using unpaired Student's t-tests comparing DEP, BA and TMT exposure groups. For all statistical calculations a $p<0.05$ was set as significant result.

\section{Conclusion}

In conclusion, our data show a robust innate fear response to TMT in GAD67 mice that is comparable to their wild type littermates, and results indicate a central role of a distinct neural circuit involving the MOB, the MeA (MeAad, MeAav, MeApv), the CeA, the LS and the BNST for the processing of TMT-related information and the regulation of innate fear behavior to TMT. Hence, we suggest that GAD67 mice are a suitable model organism to further study the role of GABAergic neurons in neuronal circuits modulating innate fear responses to TMT.

\section{References}

[1] Morrow, B.A., et al. (2000) The Predator Odor, TMT, Displays a Unique, Stress-Like Pattern of Dopaminergic and Endocrinological Activation in the Rat. Brain Research, 864, 146-151. http://dx.doi.org/10.1016/S0006-8993(00)02174-0

[2] Blanchard, R.J. and Blanchard, D.C. (1969) Crouching as an Index of Fear. Journal of Comparative and Physiological Psychology, 67, 370-375. http://dx.doi.org/10.1037/h0026779

[3] Blanchard, R.J., et al. (1990) The Characterization and Modelling of Antipredator Defensive Behavior. Neuroscience \& Biobehavioral Reviews, 14, 463-472. http://dx.doi.org/10.1016/S0149-7634(05)80069-7

[4] Endres, T., Apfelbach, R. and Fendt, M. (2005) Behavioral Changes Induced in Rats by Exposure to Trimethylthiazoline, a Component of Fox Odor. Behavioral Neuroscience, 119, 1004-1010. http://dx.doi.org/10.1037/0735-7044.119.4.1004

[5] Janitzky, K., et al. (2009) Behavioral Effects and Pattern of Brain c-fos mRNA Induced by 2,5-Dihydro-2,4,5Trimethylthiazoline, a Component of Fox Feces Odor in GAD67-GFP Knock-In C57BL/6 Mice. Behavioural Brain Research, 202, 218-224. http://dx.doi.org/10.1016/j.bbr.2009.03.038

[6] Vernet-Maury, E., Polak, E.H. and Demael, A. (1984) Structure-Activity Relationship of Stress-Inducing Odorants in the Rat. Journal of Chemical Ecology, 10, 1007-1018. http://dx.doi.org/10.1007/BF00987509

[7] Wallace, K.J. and Rosen, J.B. (2000) Predator Odor as an Unconditioned Fear Stimulus in Rats: Elicitation of Freezing by Trimethylthiazoline, a Component of Fox Feces. Behavioral Neuroscience, 114, 912-922. http://dx.doi.org/10.1037/0735-7044.114.5.912

[8] Morrow, B.A., Elsworth, J.D. and Roth, R.H. (2002) Fear-Like Biochemical and Behavioral Responses in Rats to the Predator Odor, TMT, Are Dependent on the Exposure Environment. Synapse, 46, 11-18. http://dx.doi.org/10.1002/syn.10109

[9] Nikaido, Y. and Nakashima, T. (2009) Effects of Environmental Novelty on Fear-Related Behavior and Stress Responses of Rats to Emotionally Relevant Odors. Behavioural Brain Research, 199, 241-246. http://dx.doi.org/10.1016/j.bbr.2008.11.043

[10] Rosen, J.B., West, E.A. and Donley, M.P. (2006) Not All Rat Strains Are Equal: Differential Unconditioned Fear Responses to the Synthetic Fox Odor 2,4,5-Trimethylthiazoline in Three Outbred rat Strains. Behavioral Neuroscience, 120, 290-297. http://dx.doi.org/10.1037/0735-7044.120.2.290

[11] Sotnikov, S.V., Markt, P.O., Umriukhin, A.E. and Landgraf, R. (2011) Genetic Predisposition to Anxiety-Related Behavior Predicts Predator Odor Response. Behavioural Brain Research, 225, 230-234. http://dx.doi.org/10.1016/j.bbr.2011.07.022

[12] Staples, L.G. and McGregor, I.S. (2006) Defensive Responses of Wistar and Sprague-Dawley Rats to Cat Odour and TMT. Behavioural Brain Research, 172, 351-354. http://dx.doi.org/10.1016/j.bbr.2006.04.011

[13] Uchida, T., Oki, Y., Yanagawa, Y. and Fukuda, A. (2011) A Heterozygous Deletion in the Glutamate Decarboxylase 67 Gene Enhances Maternal and Fetal Stress Vulnerability. Neuroscience Research, 69, 276-282. http://dx.doi.org/10.1016/j.neures.2010.12.010

[14] Wang, Y., et al. (2009) Fluorescent Labeling of Both GABAergic and Glycinergic Neurons in Vesicular GABA Transporter (VGAT)-Venus Transgenic Mouse. Neuroscience, 164, 1031-1043. http://dx.doi.org/10.1016/j.neuroscience.2009.09.010 
[15] Cullinan, W.E., Herman, J.P. and Watson, S.J. (1993) Ventral Subicular Interaction with the Hypothalamic Paraventricular Nucleus: Evidence for a Relay in the Bed Nucleus of the Stria Terminalis. Journal of Comparative Neurology, 332, 1-20. http://dx.doi.org/10.1002/cne.903320102

[16] Krettek, J.E. and Price, J.L. (1978) Amygdaloid Projections to Subcortical Structures within the Basal Forebrain and Brainstem in the Rat and Cat. Journal of Comparative Neurology, 178, 225-254. http://dx.doi.org/10.1002/cne.901780204

[17] Laska, M., Fendt, M., Wieser, A., Endres, T., Salazar, L.T.H. and Apfelbach, R. (2005) Detecting Danger-Or Just Another Odorant? Olfactory Sensitivity for the Fox Odor Component 2,4,5-Trimethylthiazoline in Four Species of Mammals. Physiology \& Behavior, 84, 211-215. http://dx.doi.org/10.1016/j.physbeh.2004.11.006

[18] Ayers, L.W., Asok, A., Heyward, F.D. and Rosen, J.B. (2013) Freezing to the Predator Odor 2,4,5 Dihydro 2,5 Trimethylthiazoline (TMT) Is Disrupted by Olfactory Bulb Removal but Not Trigeminal Deafferentation. Behavioural Brain Research, 253, 54-59. http://dx.doi.org/10.1016/j.bbr.2013.06.034

[19] Janitzky, K., D’Hanisc, W., Kröberc, A. and Schwegler, H. (2015) TMT Predator Odor Activated Neural Circuit in C57BL/6J Mice Indicates TMT-Stress as a Suitable Model for Uncontrollable Intense Stress. Brain Research, 1599, 1-8. http://dx.doi.org/10.1016/j.brainres.2014.12.030

[20] Curran, T. and Morgan, J.I. (1995) Fos: An Immediate-Early Transcription Factor in Neurons. Journal of Neurobiology, 26, 403-412. http://dx.doi.org/10.1002/neu.480260312

[21] Herrera, D.G. and Robertson, H.A. (1996) Activation of c-fos in the Brain. Progress in Neurobiology, 50, 83-107. http://dx.doi.org/10.1016/S0301-0082(96)00021-4

[22] Morgan, J.I. and Curran, T. (1991) Stimulus-Transcription Coupling in the Nervous System: Involvement of the Inducible Proto-Oncogenes Fos and Jun. Annual Review of Neuroscience, 14, 421-451. http://dx.doi.org/10.1146/annurev.ne.14.030191.002225

[23] Baisley, S.K., Cloninger, C.L. and Bakshi, V.P. (2011) Fos Expression Following Regimens of Predator Stress versus Footshock That Differentially Affect Prepulse Inhibition in Rats. Physiology \& Behavior, 104, 796-803. http://dx.doi.org/10.1016/j.physbeh.2011.08.001

[24] Day, H.E., Masini, C.V. and Campeau, S. (2004) The Pattern of Brain c-fos mRNA Induced by a Component of Fox Odor, 2,5-Dihydro-2,4,5-Trimethylthiazoline (TMT), in Rats, Suggests Both Systemic and Processive Stress Characteristics. Brain Research, 1025, 139-151. http://dx.doi.org/10.1016/j.brainres.2004.07.079

[25] Matsukawa, M., Imada, M., Murakami, T., Aizawa, S. and Sato, T. (2011) Rose Odor Can Innately Counteract Predator Odor. Brain Research, 1381, 117-123. http://dx.doi.org/10.1016/j.brainres.2011.01.053

[26] Pantazopoulos, H., Dolatshad, H. and Davis, F.C. (2011) A Fear-Inducing Odor Alters PER2 and c-fos Expression in Brain Regions Involved in Fear Memory. PLOS ONE, 6, e20658. http://dx.doi.org/10.1371/journal.pone.0020658

[27] Staples, L.G., Hunt, G.E., van Nieuwenhuijzen, P.S. and McGregor, I.S. (2008) Rats Discriminate Individual Cats by Their Odor: Possible Involvement of the Accessory Olfactory System. Neuroscience \& Biobehavioral Reviews, 32, 1209-1217. http://dx.doi.org/10.1016/j.neubiorev.2008.05.011

[28] Davis, M. (1998) Are Different Parts of the Extended Amygdala Involved in Fear versus Anxiety? Biological Psychiatry, 44, 1239-1247. http://dx.doi.org/10.1016/S0006-3223(98)00288-1

[29] Dielenberg, R.A., Hunt, G.E. and McGregor, I.S. (2001) "When a Rat Smells a Cat": The Distribution of Fos Immunoreactivity in Rat Brain Following Exposure to a Predatory Odor. Neuroscience, 104, 1085-1097. http://dx.doi.org/10.1016/S0306-4522(01)00150-6

[30] Endres, T. and Fendt, M. (2008) Inactivation of the Lateral Septum Blocks Fox Odor-Induced Fear Behavior. Neuroreport, 19, 667-670. http://dx.doi.org/10.1097/WNR.0b013e3282fb78d9

[31] Fendt, M., Endres, T., Lowry, C.A., Apfelbach, R. and McGregor, I.S. (2005) TMT-Induced Autonomic and Behavioral Changes and the Neural Basis of Its Processing. Neuroscience \& Biobehavioral Reviews, 29, 1145-1156. http://dx.doi.org/10.1016/j.neubiorev.2005.04.018

[32] Fendt, M., Endres, T. and Apfelbach, R. (2003) Temporary Inactivation of the Bed Nucleus of the Stria Terminalis but Not of the Amygdala Blocks Freezing Induced by Trimethylthiazoline, a Component of Fox Feces. The Journal of Neuroscience, 23, 23-28.

[33] Guthrie, K.M. and Gall, C.M. (1995) Functional Mapping of Odor-Activated Neurons in the Olfactory Bulb. Chemical Senses, 20, 271-282. http://dx.doi.org/10.1093/chemse/20.2.271

[34] Herdade, K.C., de Andrade Strauss, C.V., Zangrossi Jr., H. and de Barros Viana, M. (2006) Effects of Medial Amygdala Inactivation on a Panic-Related Behavior. Behavioural Brain Research, 172, 316-323. http://dx.doi.org/10.1016/j.bbr.2006.05.021

[35] Kobayakawa, K., et al. (2007) Innate versus Learned Odour Processing in the Mouse Olfactory Bulb. Nature, 450, 503-508. http://dx.doi.org/10.1038/nature06281 
[36] LeDoux, J.E. (1992) Brain Mechanisms of Emotion and Emotional Learning. Current Opinion in Neurobiology, 2, 191-197. http://dx.doi.org/10.1016/0959-4388(92)90011-9

[37] Li, C.-I., Maglinao, T.L. and Takahashi, L.K. (2004) Takahashi, Medial Amygdala Modulation of Predator OdorInduced Unconditioned Fear in the Rat. Behavioral Neuroscience, 118, 324-332. http://dx.doi.org/10.1037/0735-7044.118.2.324

[38] Lin da, Y., Shea, S.D. and Katz, L.C. (2006) Representation of Natural Stimuli in the Rodent Main Olfactory Bulb. Neuron, 50, 937-949. http://dx.doi.org/10.1016/j.neuron.2006.03.021

[39] Mongeau, R., Miller, G.A., Chiang, E. and Anderson, D.J. (2003) Neural Correlates of Competing Fear Behaviors Evoked by an Innately Aversive Stimulus. The Journal of Neuroscience, 23, 3855-3868.

[40] Muller, M. and Fendt, M. (2006) Temporary Inactivation of the Medial and Basolateral Amygdala Differentially Affects TMT-Induced Fear Behavior in Rats. Behavioural Brain Research, 167, 57-62. http://dx.doi.org/10.1016/j.bbr.2005.08.016

[41] Rosen, J.B. (2004) The Neurobiology of Conditioned and Unconditioned Fear: A Neurobehavioral System Analysis of the Amygdala. Behavioral and Cognitive Neuroscience Reviews, 3, 23-41. http://dx.doi.org/10.1177/1534582304265945

[42] Walker, D.L. and Davis, M. (1997) Double Dissociation between the Involvement of the Bed Nucleus of the Stria Terminalis and the Central Nucleus of the Amygdala in Startle Increases Produced by Conditioned versus Unconditioned Fear. The Journal of Neuroscience, 17, 9375-9383.

[43] Walker, D.L., Toufexis, D.J. and Davis, M. (2003) Role of the Bed Nucleus of the Stria Terminalis versus the Amygdala in Fear, Stress, and Anxiety. European Journal of Pharmacology, 463, 199-216. http://dx.doi.org/10.1016/S0014-2999(03)01282-2

[44] Wallace, K.J. and Rosen, J.B. (2001) Neurotoxic Lesions of the Lateral Nucleus of the Amygdala Decrease Conditioned Fear but Not Unconditioned Fear of a Predator Odor: Comparison with Electrolytic Lesions. The Journal of Neuroscience, 21, 3619-3627.

[45] Buron, G., Hacquemand, R., Pourie, G., Lucarz, A., Jacquot, L. and Brand, G. (2007) Comparative Behavioral Effects between Synthetic 2,4,5-Trimethylthiazoline (TMT) and the Odor of Natural Fox (Vulpes vulpes) Feces in Mice. Behavioral Neuroscience, 121, 1063-1072. http://dx.doi.org/10.1037/0735-7044.121.5.1063

[46] Endres, T. and Fendt, M. (2009) Aversion- vs Fear-Inducing Properties of 2,4,5-Trimethyl-3-Thiazoline, a Component of Fox Odor, in Comparison with Those of Butyric Acid. The Journal of Experimental Biology, 212, 2324-2327. http://dx.doi.org/10.1242/jeb.028498

[47] Hebb, A.L., Zacharko, R.M., Dominguez, H., Laforest, S., Gauthier, M., Levac, C. and Drolet, G. (2003) Changes in Brain Cholecystokinin and Anxiety-Like Behavior Following Exposure of Mice to Predator Odor. Neuroscience, 116, 539-551. http://dx.doi.org/10.1016/S0306-4522(02)00710-8

[48] Hebb, A.L., Zacharko, R.M., Gauthier, M. and Drolet, G. (2003) Exposure of Mice to a Predator Odor Increases Acoustic Startle but Does Not Disrupt the Rewarding Properties of VTA Intracranial Self-Stimulation. Brain Research, 982, 195-210. http://dx.doi.org/10.1016/S0006-8993(03)03008-7

[49] Petrovich, G.D., Canteras, N.S. and Swanson, L.W. (2001) Combinatorial Amygdalar Inputs to Hippocampal Domains and Hypothalamic Behavior Systems. Brain Research Reviews, 38, 247-289. http://dx.doi.org/10.1016/S0165-0173(01)00080-7

[50] Meredith, M. and Westberry, J.M. (2004) Distinctive Responses in the Medial Amygdala to Same-Species and Different-Species Pheromones. The Journal of Neuroscience, 24, 5719-5725. http://dx.doi.org/10.1523/JNEUROSCI.1139-04.2004

[51] Pro-Sistiaga, P., et al. (2007) Convergence of Olfactory and Vomeronasal Projections in the Rat Basal Telencephalon. Journal of Comparative Neurology, 504, 346-362. http://dx.doi.org/10.1002/cne.21455

[52] Takahashi, L.K. (2014) Olfactory Systems and Neural Circuits That Modulate Predator Odor Fear. Frontiers in Behavioral Neuroscience, 8, 72. http://dx.doi.org/10.3389/fnbeh.2014.00072

[53] Nader, K., Majidishad, P., Amorapanth, P. and LeDoux, J.E. (2001) Damage to the Lateral and Central, but Not Other, Amygdaloid Nuclei Prevents the Acquisition of Auditory Fear Conditioning. Learning Memory, 8, 156-163. http://dx.doi.org/10.1101/lm.38101

[54] Xu, H.Y., Liu, Y.-J., Xu, M.-Y., Zhang, Y.-H., Zhang, J.-X. and Wu, Y.-J. (2012) Inactivation of the Bed Nucleus of the Stria Terminalis Suppresses the Innate Fear Responses of Rats Induced by the Odor of Cat Urine. Neuroscience, 221, 21-27. http://dx.doi.org/10.1016/j.neuroscience.2012.06.056

[55] Asok, A., Ayers, L.W., Awoyemi, B., Schulkin, J. and Rosen, J.B. (2013) Immediate Early Gene and Neuropeptide Expression Following Exposure to the Predator Odor 2,5-Dihydro-2,4,5-Trimethylthiazoline (TMT). Behavioural Brain Research, 248, 85-93. http://dx.doi.org/10.1016/j.bbr.2013.03.047 
[56] Janitzky, K., Peine, A., Kröber, A., Yanagawa, Y., Schwegler, H. and Roskoden, T. (2014) Increased CRF mRNA Expression in the Sexually Dimorphic BNST of Male but Not Female GAD67 Mice and TMT Predator Odor Stress Effects upon Spatial Memory Retrieval. Behavioural Brain Research, 272, 141-149. http://dx.doi.org/10.1016/j.bbr.2014.06.020

[57] Rosen, J.B., Pagani, J.H., Rolla, K.L.G. and Davis, C. (2008) Analysis of Behavioral Constraints and the Neuroanatomy of Fear to the Predator Odor Trimethylthiazoline: A Model for Animal Phobias. Neuroscience \& Biobehavioral Reviews, 32, 1267-1276. http://dx.doi.org/10.1016/j.neubiorev.2008.05.006

[58] Kudo, T., Uchigashima, M., Miyazaki, T., Konno, K., Yamasaki, M., Yanagawa, Y., Minami, M. and Watanabe, M. (2012) Three Types of Neurochemical Projection from the Bed Nucleus of the Stria Terminalis to the Ventral Tegmental Area in Adult Mice. The Journal of Neuroscience, 32, 18035-18046. http://dx.doi.org/10.1523/JNEUROSCI.4057-12.2012

[59] Sajdyk, T., Johnson, P.L., Fitz, S.D. and Shekhar, A. (2008) Chronic Inhibition of GABA Synthesis in the Bed Nucleus of the Stria Terminalis Elicits Anxiety-Like Behavior. Journal of Psychopharmacology, 22, 633-641. http://dx.doi.org/10.1177/0269881107082902

[60] Sun, N. and Cassell, M.D. (1993) Intrinsic GABAergic Neurons in the Rat Central Extended Amygdala. Journal of Comparative Neurology, 330, 381-404. http://dx.doi.org/10.1002/cne.903300308

[61] Bali, B., Erdélyi, F., Szabó, G. and Kovács, K.J. (2005) Visualization of Stress-Responsive Inhibitory Circuits in the GAD65-eGFP Transgenic Mice. Neuroscience Letters, 380, 60-65. http://dx.doi.org/10.1016/j.neulet.2005.01.014

[62] Davis, M. and Shi, C. (1999) The Extended Amygdala: Are the Central Nucleus of the Amygdala and the Bed Nucleus of the Stria Terminalis Differentially Involved in Fear versus Anxiety? Annals of the New York Academy of Sciences, 877, 281-291. http://dx.doi.org/10.1111/j.1749-6632.1999.tb09273.x

[63] Egli, R.E. and Winder, D.G. (2003) Dorsal and Ventral Distribution of Excitable and Synaptic Properties of Neurons of the Bed Nucleus of the Stria Terminalis. Journal of Neurophysiology, 90, 405-414. http://dx.doi.org/10.1152/jn.00228.2003

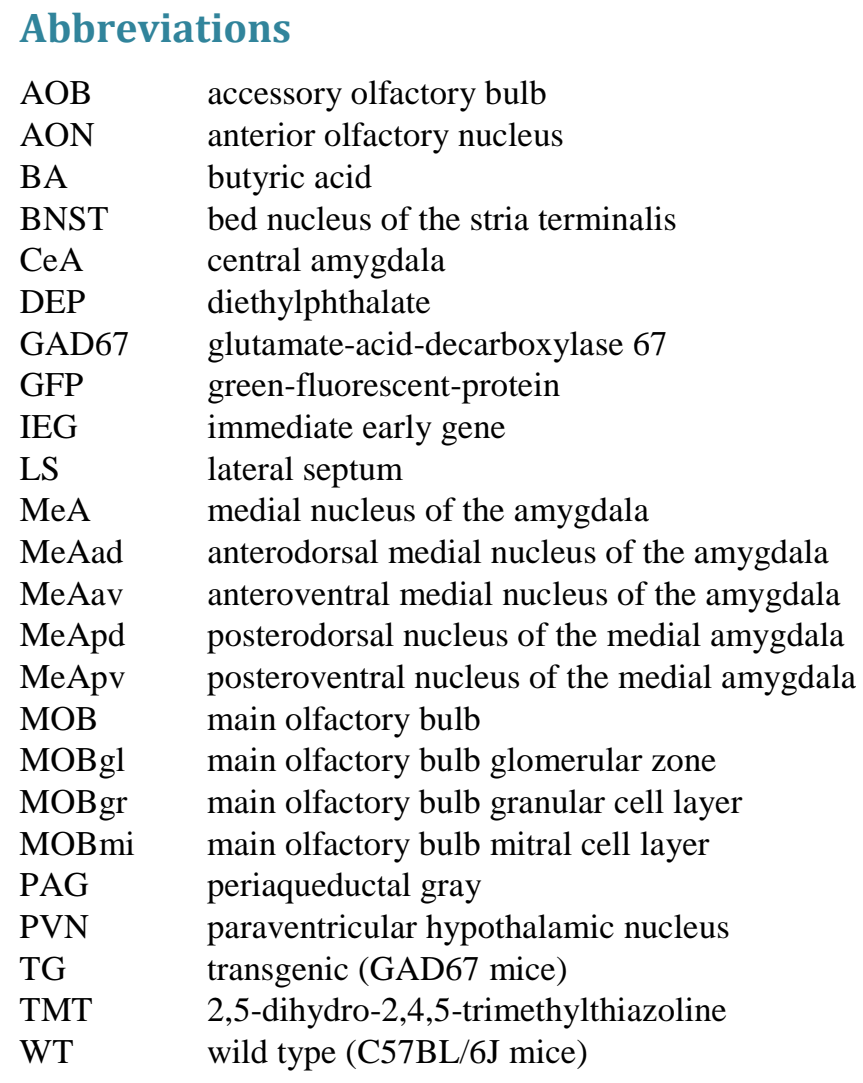

